\title{
The purple urine bag syndrome
}

\author{
Pauline Vanderweckene ${ }^{1}$, Christiane Counasse ${ }^{2}$ and Jean-Marie H Krzesinski ${ }^{*}$ \\ ${ }^{1}$ Department of Nephrology, University of Liège, Belgium \\ ${ }^{2}$ Revalidation unit CHU Liège, University of Liège, Belgium
}

\section{Case}

An 81-year-old man was hospitalized for a long time in geriatric unit for a meningeal hemorrhage with secondary hydrocephalus. $\mathrm{He}$ underwent a ventriculoperitoneal shunt but had limited mobility with confusion and needed chronic urinary tract catheter. He has mainly cardiovascular history (coronary bypass, ventricular arrhythmia, hypertension, atril fibrillation and pace make). During hospitalization, his chronic kidney disease has increased (eGFR $30 \mathrm{~mL} / \mathrm{min}$ ) and he has presented constipation requiring laxative medication. One day, the health care team noticed the purple coloration of the urine bag (Figure 1). A urinalysis showed pyuria, and an alkaline $\mathrm{pH}(\mathrm{pH}$ 8). The urine culture yielded E. Coli and K. Pneumoniae (growth was greater than $10^{6} \mathrm{CFU} / \mathrm{mL}$ for both). The purple urine bag syndrome was diagnosed.

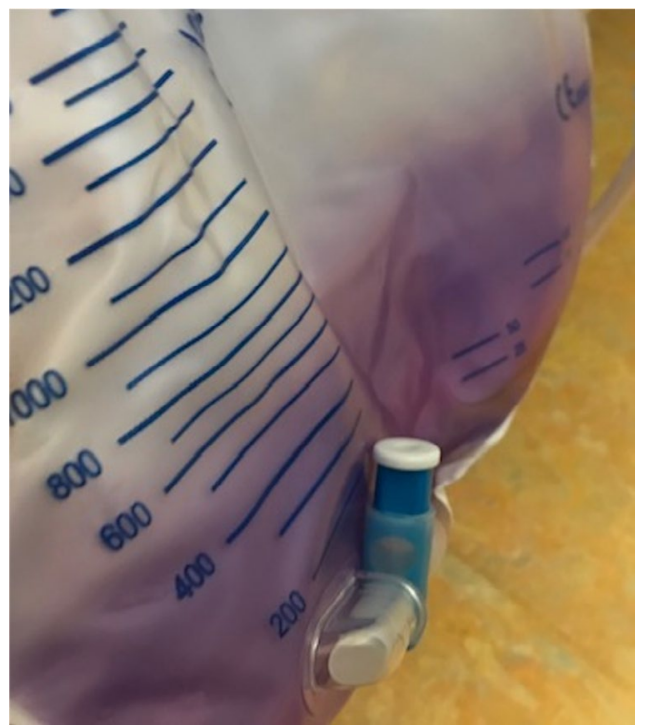

\section{Discussion}

This syndrome is sometimes observed in cases of urinary infection in bed-ridden patients with long-term indwelling urinary catheters, constipation and alkaline urinary $\mathrm{pH}[1]$.

It was first described in 1978 and the mechanism only understood in 1988 [2].

The purple urine bag syndrome is linked to intestinal bacterial overgrowth, secondary to constipation, with an increase of dietary tryptophan metabolism into indol products absorbed and conjugated to indoxyl sulfate metabolites in the liver, then excreted in the urine with a transformation into indoxyl by sulfatases and phosphatases produced by urinary bacteria, these include Escherichia Coli, Proteus Mirabilis, Morganellla morganii, Klebsiella pneumoniae, Providencia stuartii, Providencia rettgeri et Pseudomonas Aeruginosa). The indoxyl products are then converted into indigo (blue) and indirubin (red) metabolites, the mixture of which causes the purple color in the plastic urine bag.

The prognosis is usually good, depending on proper management of infection [3].

\section{References}

1. Su K (2005) Case analysis of purple urine-bag syndrome at a long-term care service in a community hospital. Chang Gung Med J 28: 636-642.

2. Dealer SF (1988) Enzymatic degradation of urinary indoxyl sulfate by Providencia stuartii and Klebsiella pneumoniae causes the purple urine bag syndrome. $J$ Clin Microbiol 26: 2152-2156.

3. Su, Wang (2019) Risk factors of mortality in patients with purple urine bag syndrome. Journal of Drug Assessment 8: 21-24.

Figure 1. Purple color of the urine in the plastic bag collector

Copyright: (C2020 Vanderweckene P. This is an open-access article distributed under the terms of the Creative Commons Attribution License, which permits unrestricted use, distribution, and reproduction in any medium, provided the original author and source are credited.
${ }^{\star}$ Correspondence to: Jean-Marie Krzesinski, Department of Nephrology, CHU Liège B35 4000 Liege, Belgium, E-mail: jm.krzesinski@chuliege.be

Received: January 27, 2020; Accepted: February 20, 2020; Published: February 24,2020 